\title{
Review Article NADPH Oxidases in Chronic Liver Diseases
}

\author{
Joy X. Jiang and Natalie J. Török \\ Department of Internal Medicine, Division of Gastroenterology and Hepatology, UC Davis Medical Center, 4150 V Street, \\ Sacramento, CA 95817, USA
}

Correspondence should be addressed to Joy X. Jiang; xiaosong.jiang@ucdmc.ucdavis.edu and Natalie J. Török; natalie.torok@ucdmc.ucdavis.edu

Received 15 July 2014; Accepted 30 October 2014; Published 30 November 2014

Academic Editor: Jose J. Marin

Copyright (C) 2014 J. X. Jiang and N. J. Török. This is an open access article distributed under the Creative Commons Attribution License, which permits unrestricted use, distribution, and reproduction in any medium, provided the original work is properly cited.

Oxidative stress is a common feature observed in a wide spectrum of chronic liver diseases including viral hepatitis, alcoholic, and nonalcoholic steatohepatitis. The nicotinamide adenine dinucleotide phosphate (NADPH) oxidases (NOXs) are emerging as major sources of reactive oxygen species (ROS). Several major isoforms are expressed in the liver, including NOX1, NOX2, and NOX4. While the phagocytic NOX2 has been known to play an important role in Kupffer cell and neutrophil phagocytic activity and inflammation, the nonphagocytic NOX homologues are increasingly recognized as key enzymes in oxidative injury and wound healing. In this review, we will summarize the current advances in knowledge on the regulatory pathways of NOX activation, their cellular distribution, and their role in the modulation of redox signaling in liver diseases.

\section{Introduction}

In chronic liver diseases, hepatocyte injury triggers Kupffer cell activation and hepatic stellate cell (HSC) transdifferentiation to matrix-producing myofibroblasts, and the accumulation of extracellular matrix leads to fibrosis and cirrhosis $[1,2]$. Most if not all pathogenic insults in the liver can cause oxidative stress, inducing lipid peroxidation, protein oxidation, and DNA damage, leading to hepatocyte mitochondrial dysfunction, amplifying inflammation and initiating fibrosis [3]. As important second messengers, ROS can have an impact on cell death/survival pathways [4]. In the liver there are several important sources of ROS production. While the role of cytochrome P4502E1 (CYP2E1), the mitochondrial respiratory chain, arachidonic acid oxidation, and the xanthine oxidase system have been extensively studied in the past [5-7], recently the group of NOX enzymes have been emerging as major sources of ROS production. $\mathrm{H}_{2} \mathrm{O}_{2}$ is one of the main oxidative radicals during liver diseases and it is generated by the NADPH oxidases or complex III of the mitochondrial respiratory chain. In physiological situations the amount of $\mathrm{H}_{2} \mathrm{O}_{2}$ is under a tight control by the peroxiredoxins and glutathione peroxidases as well as by catalase [8]. During chronic liver injury however this balance is perturbed and parenchymal cells are exposed to increasing concentrations of ROS. Among the seven NOX homologues found in mammals (NOX1, NOX2, NOX3, NOX4, NOX5, DUOX1, and DUOX2), the main ROS-producing NOXs in the liver are NOX1, NOX2, and NOX4 [9] (Table 1). NOX1 and 2 are mainly producing superoxide whereas NOX4 directly produces $\mathrm{H}_{2} \mathrm{O}_{2}$. To form active complexes, NOX1 and NOX2 bind with their structural subunits: NOX1 associates with p22phox, p47phox, p67phox, and active Rac (or NOXO1 and NOXA1, the homologues of p47phox and p67phox); besides these subunits, NOX2 activation also requires p40phox [10]. NOX4 is a constitutively active membrane-bound isoform and according to current knowledge associates with p22phox, only [10] (Figure 1). Thus interaction between the subunits is an important determinant of enzyme activity [11].

In general, to maintain homeostasis free radicals are scavenged by a powerful antioxidant system in the liver composed of enzymatic and nonenzymatic molecules. The former group consists of superoxide dismutases (SOD), catalase, and enzymes regulating glutathione (GSH) synthesis. The nonenzymatic antioxidants include the coenzyme Q10, GSH, and ROS binding proteins such as thioredoxin (Trx) [1214]. In response to oxidative stress initially there is a rapid induction of antioxidant signaling. The transcription factor 
TABLE 1: The major NOX homologues expressed in the liver and their function.

\begin{tabular}{lll}
\hline NOX homologue & Expressing cells in the liver & Function \\
\hline NOX1 & $\begin{array}{l}\text { HSC, hepatocytes, sinusoidal endothelial cells (SECs), } \\
\text { and macrophages }\end{array}$ & $\begin{array}{l}\text { HSC: liver fibrogenesis [18, 19], proliferation [20], } \\
\text { senescence [19] } \\
\text { Hepatocyte: HCV-mediated oxidative stress [21] } \\
\text { Macrophage: oxidative stress, liver fibrogenesis [18] } \\
\text { SECs: unknown }\end{array}$ \\
\hline NOX2 & Macrophages, HSC, SECs, and hepatocytes & $\begin{array}{l}\text { Macrophage: oxidative burst [10], liver fibrosis [22] } \\
\text { HSC: phagocytosis [18], liver fibrosis [18, 22-25] } \\
\text { Hepatocyte: unknown } \\
\text { SECs: unknown }\end{array}$ \\
\hline NOX3 & HepG2 & Unknown \\
\hline NOXn & Hepatocytes, HSC, and SECs & $\begin{array}{l}\text { Hepatocyte: oxidative stress [21, 26], apoptosis [27-30] } \\
\text { HSC: liver fibrogenesis [28, 29] } \\
\text { SECs: unknown }\end{array}$ \\
\hline Duox1 & Unknown & \\
\hline Duox2 & Unknown & \\
\hline
\end{tabular}
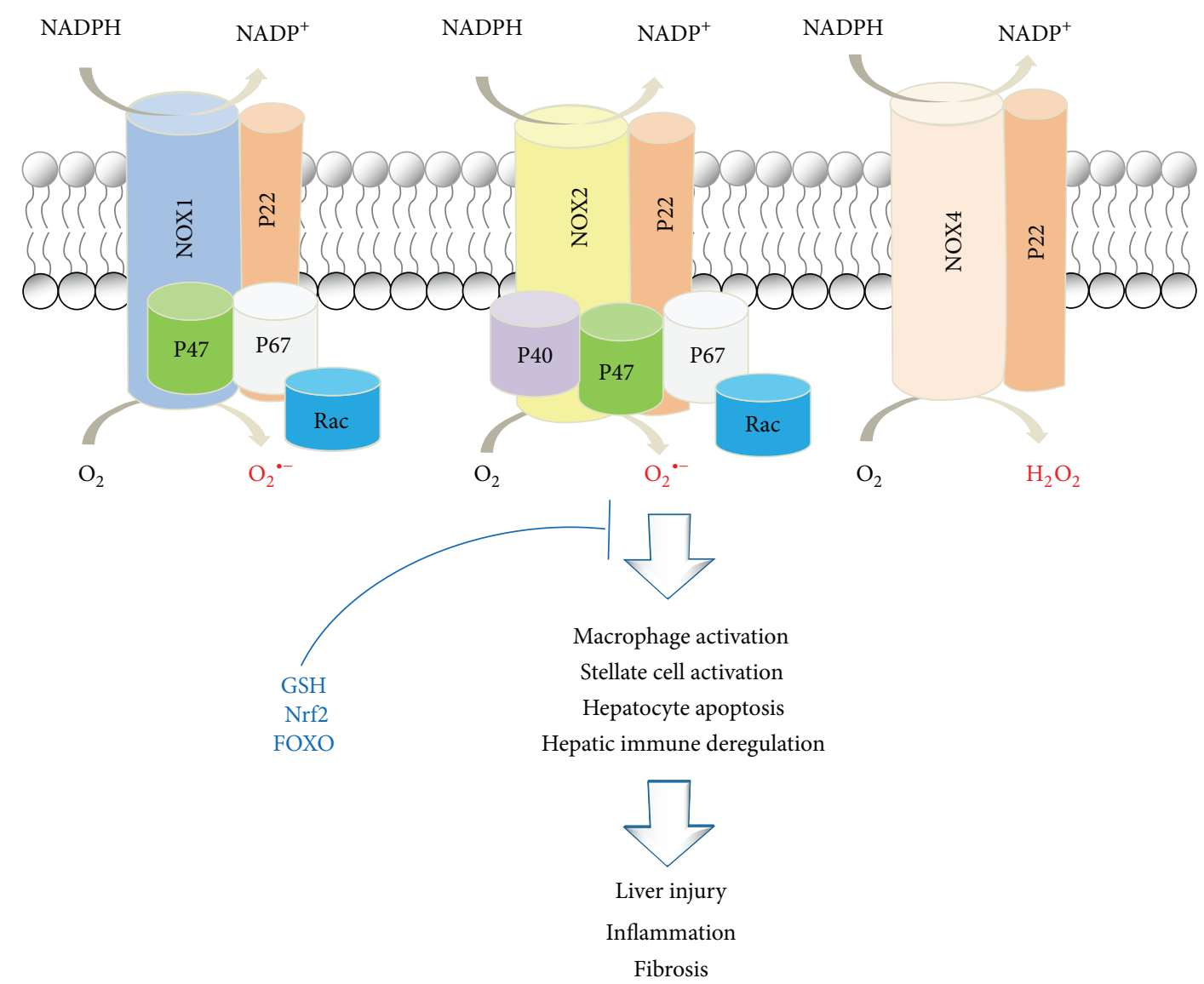

FIGURE 1: NADPH oxidases in the liver. The NOX isoforms expressed in the liver are NOX1, NOX2, and NOX4. NOX1 and NOX2 form multimeric complexes on the plasma membrane with their regulatory components and catalyze the production of superoxide $\left(\mathrm{O}_{2}{ }^{\cdot-}\right)$ whereas NOX4 is constitutively active and mainly produces $\mathrm{H}_{2} \mathrm{O}_{2}$. The NOX-derived reactive oxygen species (ROS) mediate hepatocyte apoptosis, macrophage, and stellate cell activation in a spectrum of liver diseases. 
nuclear factor erythroid 2-related factor 2 (Nrf2) is a master regulator inducing the transcription of a spectrum of genes related to GSH metabolism via the antioxidant responsive element (ARE) on the target genes and is also playing a role in xenobiotic detoxification and proteome maintenance [15]. Another group of transcription factors the Forkhead box $\mathrm{O}(\mathrm{FOXO})$ regulate the SOD and catalase transcripts [16, 17]. However, after prolonged chronic injury, the antioxidant responses gradually fail leading to the unopposed actions of ROS inflammation and fibrogenesis.

\section{Hepatitis C}

The role of NOXs in the pathogenesis of HCV has been increasingly recognized involving both the dysregulation of T-cell responsiveness and hepatocyte injury. The NS3 viral protein was shown to induce a significant ROS production in monocytes which coincided with p47phox phosphorylation and translocation [31]. The NS3-activated phagocytes, in turn, could induce the dysfunction of $\mathrm{CD} 3+/ 56-\mathrm{T}$ cells, CD3-/ $56+$ natural killer (NK) cells, and CD3+/56+ NKT cells [32]. HCV could also promote the recruitment of CD33+ myeloid-derived suppressor cells leading to the NOX2/ROSmediated suppression of T-cell responsiveness thus leading to persistent HCV infection [26]. The nonphagocytic NOXs were shown to be instrumental in HCV-induced oxidative stress. In hepatocytes, HCV proteins directly induced NOX4 expression through an autocrine TGF $\beta$-dependent mechanism [21]. In a study by de Mochel et al., both NOX1 and 4 were localized in the nucleus of HCV-infected hepatocytes and were responsible for nuclear ROS production and nitrotyrosine generation [33]. The induction of NOX4 was believed to be mediated by TGF $\beta /$ Smad signaling [21, 33]. In patients with HCV, using losartan "an AT1 receptor blocker" the expression of NOX activator 1 (NOXA-1) and organizer 1 (NOXO-1), Rac-1 and fibrogenic genes were downregulated [34].

\section{Alcoholic and Nonalcoholic Steatohepatitis (ASH and NASH)}

Oxidative stress plays a key role in both ASH and NASH [35]. The phagocytic NOX in Kupffer cells is thought to be essential in the pathogenesis of early alcohol-induced hepatitis by activating NF- $\kappa \mathrm{B}$, which in turn activates the production of cytotoxic TNF $\alpha[36,37]$. This was later also confirmed in the studies of Cubero and Nieto where chronic ethanol administration and arachidonic acid (AA) synergistically mediated Kupffer cell activation via an NADPH oxidasedependent mechanism [38]. In a different study, chronic ethanol feeding increased the LPS-stimulated NADPH oxidase-dependent production of ROS in Kupffer cells, and ERK1/2 was an important target of ROS leading to an enhanced LPS-stimulated TNF $\alpha$ production [39]. In hepatocytes the p47phox subunit containing NOXs seemed to play an important role in alcoholic steatohepatitis with a mechanism involving the lipid droplet-stabilizing protein, adipocyte differentiation-related protein (ADRP), and the fatty acid synthesis-associated genes, fatty acid synthase (FASN), and
acetyl-CoA carboxylase (ACACA) [40]. NOXs and HIF- $1 \alpha$ were shown to be involved in the alcohol-mediated induction of endothelin-1 (ET-1) in the liver sinusoidal endothelial cells (LSEC). This effect was attenuated by transfection of the p47phox siRNA suggestive of the activation of either NOX1 or 2 in these cells [41].

There are several lines of evidence suggesting that NOXs are also significant sources of oxidative radicals in NASH [42, 43]. Metabolic syndrome and diabetes mellitus are amongst the strongest risk factors for progressive NASH leading to liver fibrosis and cirrhosis. Persistent hyperglycemia can stimulate HSC to proliferate and produce extracellular matrix through activation of NOXs [43-45]. Increased NOX activity was observed in $f a / f a$ rats on high fat diet and this was correlated to an increase in lipid peroxidation [46]. However, it has not been fully understood as to which NOX homologue is crucial to disease progression in ASH/NASH. Activation of the phagocytic NOX2 in Kupffer cells, for example, is an important determinant of leptin-mediated production of oxidative radicals and macrophage activation [47]. Leptinmediated induction of NADPH oxidase activity in HSC is also an important factor in fibrosis progression in NASH $[48,49]$, and leptin was shown to induce collagen $1 \alpha(\mathrm{I})$ by a NOX and Sp1- and Sp3-mediated pathway [50]. Advanced glycation end-products that accumulate in patients with diabetes were described to have a major contribution to tissue injury by the activation of the receptor for AGEs (RAGE) and subsequent release of ROS [23, 51]. We recently showed that RAGE activation is directly linked to the activation of NOX2 in the liver causing a downregulation of TIMP3 levels and hence an unopposed increase in TACE and TNF $\alpha$ activity leading to increased injury and fibrosis in two different dietary models of NASH [24]. The NOX2 $2^{-/-}$mice in this study developed attenuated fibrosis but steatosis was unchanged consistent with the findings in the $447 \mathrm{phox}^{-/-}$and $\mathrm{NOX}^{-1-}$ mice on MCD diet $[25,52]$, suggesting that NOX2 regulates fibrosis but not steatosis. The dominant NOX homologue in hepatocytes responsible for redox stress signaling in NASH remains to be identified. As hepatocyte cell death is a major propelling factor for fibrogenesis in NASH [53], it is very plausible that one or several NOX homologues play a major role in hepatocyte ER stress. For example, CD95L was shown to induce oxidative stress in hepatocytes via NOX1 or 2 involving ceramide and $\mathrm{PKC} \zeta$ [27]. At the same time NOX4 activation in hepatocytes was shown to be proapoptotic in different conditions $[28,29,54]$ and thus likely to contribute to NASH progression. It is also important to note that NOXs could potentially confer an individual sensitivity to metabolic syndrome and NASH by polymorphisms associated with ROS generation. Recently it was shown that a single nucleotide polymorphism, rs1836882 in the NOX4 gene's promoter region, was associated higher caloric intake and ROS levels [22].

\section{Liver Fibrosis}

Liver fibrosis is a result of a complex interplay between several cell types during chronic liver injury leading to HSC 
activation and deposition of scar tissue. It is more and more recognized that NOXs play a significant role in this process. The key role of NOX2 activation in macrophages has been explored by Paik et al. [18]. Using NOX2 bone marrow (BM) chimeric mice, they showed that the $\mathrm{NOX}^{-/-} \mathrm{BM}$ cells significantly reduced fibrosis in wt. recipient mice treated with $\mathrm{CCl} 4$ [18]. HSC also express functional NOX2, and the enzyme could be activated by the phagocytosis of apoptotic hepatocytes triggering the upregulation of $\alpha \mathrm{SMA}$, collagen I, and TGF $\beta$ as shown by our group [20]. NOX2-derived ROS could directly activate collagen I transcription in HSC as the promoter activity was significantly suppressed in $\mathrm{NOX}^{-/-}$cells or cells transfected with truncated promoter constructs, lacking the ROS-responsive area. Consistently, $\mathrm{NOX}^{-/-}$mice showed attenuated fibrosis in response to bile duct ligation (BDL) [20] or CCl4 injection [18].

Hepatocytes and sinusoidal endothelial also express all NOX2 subunits; however, the mechanism of enzyme activation or its functional relevance in these cells is yet to be determined.

NOX1 and NOX4 are widely expressed in the liver, mainly by hepatocytes, HSC, and endothelial cells [55]. NOX1 was upregulated in fibrotic livers and in active $\mathrm{HSC}$, and $\mathrm{NOX}^{-/-}$ mice developed attenuated fibrosis after $\mathrm{CCl} 4$ injection or BDL $[18,19]$. NOX1 could be induced by PDGF leading to HSC proliferation [19]. Angiotensin II is also a potent inducer of ROS production via NOXs, and liver fibrosis and the expression of procollagen $\alpha 1(\mathrm{I}), \mathrm{TGF} \beta$, and Timpl were attenuated in the $\mathrm{p} 47 \mathrm{phox}^{-/-}$mice [56]. Later, it was shown that both NOX1 and NOX2 were involved in angiotensin II signaling as HSC deficient in these NOXs to upregulate collagen $\alpha 1$ (I) and TGF $\beta$ in response to angiotensin II [18]. Interestingly, recently it was found that CCN1, a matricellular protein secreted by hepatocytes could engage integrin $\alpha 6 \beta 1$ to induce ROS accumulation in HSC and portal fibroblasts through the Racl-NOX1 complex regulating HSC senescence [30]. Thus the role of NOX1 may be context or disease stage dependent, and further studies would be required to determine the regulation of its function during disease progression.

Different from the other NOXs, NOX4 is constitutively active associated with $\mathrm{p} 22$ phox while other regulatory subunits are not known to be required [57]. NOX4 is induced in patients with HCV [28] and NASH (unpublished observations). TGF $\beta$-mediated NOX4 activation in hepatocytes is proapoptotic [58] and is required for FasL or TNF $\alpha$ mediated apoptosis [29], therefore indirectly contributing to fibrogenesis. NOX4 is increasingly induced in HSC during culture-activation, and $\mathrm{NOX} 4^{-/-}$cells have blunted collagen I production $[28,29]$.

So far, all examined NOXs displayed profibrogenic activity both in vitro and in vivo. Further studies are required, however, to determine whether there is a functional redundancy among the different NOX homologues, disease stage-specific function, or whether there is a compensatory increase (or decrease) in their function in the different knockout models. These data will be required to design adequate antifibrotic therapies that have no significant off-target effects. To date, a dual NOX4/1 inhibitor GKT137831 has shown promise in several studies [29, 59]. Using this inhibitor Aoyama and colleagues demonstrated a protective effect in CCl4-treated SOD1 active mutant transgenic mice that developed exacerbated fibrosis [59]. In the BDL model of fibrosis mice in both the preventive and treatment arm displayed significantly improved liver injury and fibrosis [29]. Our unpublished data also showed that GKT137831 improved fibrosis in mice fed with NASH-inducing diets.

\section{HCC}

The role of different NOX homologues in the development of hepatocellular carcinoma (HCC) is still under investigation. Recent evidence suggests that NOX4 may be protective in HCC, as TGF- $\beta$-induced liver tumor cell senescence was NOX4-dependent [60]. NOX4 expression was suppressed in human and mouse HCC. Silencing NOX4 in immortalized HCC cells promoted proliferation and xenograft experiments in athymic mice indicating that NOX4 silencing conferred an advantage to HCC growth, resulting in earlier onset of tumor formation and increase in tumor size [61]. In a different study the tumor suppressor STAT5 ${ }^{-/-}$mice developed severe hepatic steatosis as well as hepatocellular carcinomas by 17 months of age, and the authors showed that one of the target genes of STAT5 was NOX4. STAT5-induced activation of the proapoptotic proteins Puma and Bim was dependent on NOX4 [54].

On the other hand, NOX1 is considered as a survival signal in tumor cells. The epidermal growth factor receptor(EGFR-) mediated HCC cell growth was mediated by NOX1 and p38/Akt activation. NOX1 siRNA inhibited the expression of EGFR, TGF $\alpha$, and consequently cell proliferation in an autocrine manner [62]. The tumor suppressor HACE1 (HECT domain and Ankyrin repeat Containing E3 ubiquitin-protein ligase 1) ubiquitinates and degrades active Racl thereby controlling Racl-dependent NADPH oxidase activation. HACE1 deletion caused chronic oxidative stress, DNA damage, and enhanced tumor growth, and the effects of HACE1 deficiency were reversed in $\mathrm{NOX1}^{-/-}$cells [63]. Overall, it is still early to postulate whether different NOXs have differential effects on HCC tumor initiation, proliferation, or metastatic capacity. Further in vivo studies using inducible knockouts may be needed to advance our knowledge on the role of NOXs in hepatocarcinogenesis. The prolonged use of potential fibrosis inhibitors such as NO1/4, however, may require caution in late-stage liver diseases.

\section{Antioxidant Signals}

Glutathione (GSH), the nonprotein ROS scavenger, plays a major protective role in liver injury [13]. The amount of GSH is tightly related to the cellular oxidative homeostasis, which is mediated by a transcription factor nuclear factor erythroid 2-related factor 2 (Nrf2). As a part of defensive response to liver injury, Nrf2 dissociates from its inhibitory regulator Kelch-like ECH associating protein 1 (Keapl) in response to oxidative stress and translocates to nucleus 
to induce the transcription of antioxidant defense genes GSH synthetases (Gclc and Gclm), glutathione-S-transferase (Gst), GSH peroxidase (GPx), GSH reductase (GR), and $\mathrm{NAD}(\mathrm{P}) \mathrm{H}$ quinone oxidoreductase 1 (Nqo1), [15, 64]. Nrf2 has been shown to be protective against liver injury induced by multiple pathogenic stimuli [65]. Cell line studies demonstrated that Nrf2 was induced by hepatitis B and hepatitis C; and knockdown Nrf2 by siRNA lowered the cell survival rate $[66,67]$. Studies on Nrf2 null mice and Keap1 knockout mice proved that Nrf2 was activated in chronic alcohol consumption by CYP2E1, and that it played a protective role $[68,69]$. Nrf2 was shown to be in acute liver injury induced by various hepatotoxicants including $\mathrm{CCl} 4$, ethanol, and acetaminophen [70]. Nrf2 knockout mice were more sensitive to diet-induced steatosis, inflammation, and fibrosis [71, 72]; and Nrf2 pharmaceutical activators were beneficial to rats on high fat diet [73]. However, in transgenic mice expressing constitutively activated Nrf2, no protective effects were found against CCl4-induced liver injury and fibrosis and regeneration was impaired, too. This was thought to be due to the induction of cyclin-dependent kinase inhibitor P15 and proapoptotic protein Bim [74]. A recent study in lung fibrosis demonstrated the importance of NOX4-Nrf2 balance in redox homeostasis. Comparing young and aged mice NOX4 in aging mice had an impaired ability to induce Nrf2 responses and were more prone to develop persistent fibrosis [75].

The Forkhead box gene, group $\mathrm{O}$ (FOXO) family of transcription factors are important metabolic and antioxidant regulators and are known to play a complex role in liver diseases [76]. Their activity is tightly regulated by posttranslational modifications, including phosphorylation or acetylation [77]. It is well established that activation of FOXO transcription factors reduces the level of oxidative stress by the induction of enzymes that breakdown ROS such as MnSOD and catalase [78]. FOXO1 can directly induce MnSOD in HSC and inhibit HSC proliferation, and the FoxO1 (+/-) mice were more susceptible to the BDL-induced fibrosis [79]. FOXO1 is also a direct transcriptional regulator of gluconeogenesis by inducing the phosphoenolpyruvate carboxykinase (PEPCK)/glucose 6-phosphate pathway and disrupting mitochondrial metabolism and lipid metabolism via heme oxygenase $1 /$ sirtuin $1 / \operatorname{Ppar} \gamma \mathrm{cl} \alpha$ pathway, thereby likely playing a significant role in NASH $[76,80,81]$. FOXO3 were shown to regulate the innate immune signaling pathway in HCV directly suppressing TLR signaling [82]. It is also important to note that in certain context FoxO3 could mediate oxidative stress-induced apoptosis of hepatocytes. In the hepatotoxin 3,5-diethoxycarbonyl-1,4-dihydrocollidine (DDC) diet model Wnt/ $\beta$-catenin signaling was required for hepatocyte protection against oxidative stress-induced apoptosis through the inhibition of FoxO3 [83].

\section{Conclusion}

Oxidative stress is an important driving force in almost all chronic liver diseases. Although hepatocytes are equipped with potent adaptive antioxidant mechanisms to maintain homeostasis the persistent imbalance between the generation of ROS and the antioxidant defense capacity of hepatocytes can cause cell death, inflammation, and fibrosis. Phagocytic and nonphagocytic NADPH oxidases are major sources of ROS in liver diseases and play key roles in hepatocyte damage and HSC/KC activation; therefore they have become attractive therapeutic targets against chronic liver diseases and fibrosis. Further studies are, however, required to carefully analyze the cell and stage specific regulation of NOX activity in liver disease to be able to translate the data for human trials and to design adequate and rational treatments.

\section{Conflict of Interests}

The authors declare that there is no conflict of interests regarding the publication of this paper.

\section{References}

[1] Y. Lee and S. L. Friedman, "Fibrosis in the liver: acute protection and chronic disease," Progress in Molecular Biology and Translational Science, vol. 97, pp. 151-200, 2010.

[2] J. X. Jiang and N. J. Torok, "Liver injury and the activation of the hepatic myofibroblasts," Current Pathobiology Reports, vol. 1, pp. 215-223, 2013.

[3] V. Sánchez-Valle, N. C. Chávez-Tapia, M. Uribe, and N. Méndez-Sánchez, "Role of oxidative stress and molecular changes in liver fibrosis: a review," Current Medicinal Chemistry, vol. 19, no. 28, pp. 4850-4860, 2012.

[4] J. X. Jiang, K. Mikami, S. Venugopal, Y. Li, and N. J. Török, "Apoptotic body engulfment by hepatic stellate cells promotes their survival by the JAK/STAT and Akt/NF- $\kappa$ B-dependent pathways," Journal of Hepatology, vol. 51, no. 1, pp. 139-148, 2009.

[5] H. Jaeschke, "Reactive oxygen and mechanisms of inflammatory liver injury: present concepts," Journal of Gastroenterology and Hepatology, vol. 26, supplement 1, pp. 173-179, 2011.

[6] C. S. Lieber, "Role of oxidative stress and antioxidant therapy in alcoholic and nonalcoholic liver diseases," Advances in Pharmacology, vol. 38, pp. 601-628, 1997.

[7] A. I. Cederbaum, "Iron and CYP2E1-dependent oxidative stress and toxicity," Alcohol, vol. 30, no. 2, pp. 115-120, 2003.

[8] H. Sies, "Role of metabolic $\mathrm{H}_{2} \mathrm{O}_{2}$ generation: redox signaling and oxidative stress," Journal of Biological Chemistry, vol. 289, no. 13, pp. 8735-8741, 2014.

[9] Y.-H. Paik, J. Kim, T. Aoyama, S. de Minicis, R. Bataller, and D. A. Brenner, "Role of NADPH oxidases in liver fibrosis," Antioxidants \& Redox Signaling, vol. 20, no. 17, pp. 2854-2872, 2014.

[10] J. D. Lambeth, "NOX enzymes and the biology of reactive oxygen," Nature Reviews Immunology, vol. 4, no. 3, pp. 181-189, 2004.

[11] A. Mizrahi, Y. Berdichevsky, Y. Ugolev et al., "Assembly of the phagocyte NADPH oxidase complex: chimeric constructs derived from the cytosolic components as tools for exploring structure-function relationships," Journal of Leukocyte Biology, vol. 79, no. 5, pp. 881-895, 2006.

[12] J. I. Cohen, X. Chen, and L. E. Nagy, "Redox signaling and the innate immune system in alcoholic liver disease," Antioxidants and Redox Signaling, vol. 15, no. 2, pp. 523-534, 2011.

[13] S. C. Lu, "Glutathione synthesis," Biochimica et Biophysica Acta, vol. 1830, no. 5, pp. 3143-3153, 2013. 
[14] L. Yuan and N. Kaplowitz, "Glutathione in liver diseases and hepatotoxicity," Molecular Aspects of Medicine, vol. 30, no. 1-2, pp. 29-41, 2009.

[15] T. W. Kensler, N. Wakabayashi, and S. Biswal, "Cell survival responses to environmental stresses via the Keap1-Nrf2-ARE pathway," Annual Review of Pharmacology and Toxicology, vol. 47, pp. 89-116, 2007.

[16] G. J. Kops, T. B. Dansen, P. E. Polderman et al., "Forkhead transcription factor FOXO3a protects quiescent cells from oxidative stress," Nature, vol. 419, no. 6904, pp. 316-321, 2002.

[17] A. I. Malik and K. B. Storey, "Transcriptional regulation of antioxidant enzymes by FoxO1 under dehydration stress," Gene, vol. 485, no. 2, pp. 114-119, 2011.

[18] Y. H. Paik, K. Iwaisako, E. Seki et al., “The nicotinamide adenine dinucleotide phosphate oxidase (NOX) homologues NOX1 and NOX2/gp91(phox) mediate hepatic fibrosis in mice," Hepatology, vol. 53, no. 5, pp. 1730-1741, 2011.

[19] W. Cui, K. Matsuno, K. Iwata et al., "NOX1/nicotinamide adenine dinucleotide phosphate, reduced form (NADPH) oxidase promotes proliferation of stellate cells and aggravates liver fibrosis induced by bile duct ligation," Hepatology, vol. 54, no. 3, pp. 949-958, 2011.

[20] J. X. Jiang, S. Venugopal, N. Serizawa et al., "Reduced nicotinamide adenine dinucleotide phosphate oxidase 2 plays a key role in stellate cell activation and liver fibrogenesis in vivo," Gastroenterology, vol. 139, no. 4, pp. 1375.e4-1384.e4, 2010.

[21] H. E. Boudreau, S. U. Emerson, A. Korzeniowska, M. A. Jendrysik, and T. L. Leto, "Hepatitis C virus (HCV) proteins induce NADPH oxidase 4 expression in a transforming growth factor $\beta$-dependent manner: a new contributor to HCV-induced oxidative stress," Journal of Virology, vol. 83, no. 24, pp. 12934-12946, 2009.

[22] Q. Liu, H. Li, N. Wang et al., "Polymorphism of rs1836882 in NOX4 gene modifies associations between dietary caloric intake and ROS levels in peripheral blood mononuclear cells," PLoS ONE, vol. 8, no. 12, Article ID e85660, 2013.

[23] H. Vlassara and J. Uribarri, "Advanced glycation end products (AGE) and diabetes: cause, effect, or both?" Current Diabetes Reports, vol. 14, no. 1, article 453, 2014.

[24] J. X. Jiang, X. Chen, H. Fukada, N. Serizawa, S. Devaraj, and N. J. Török, "Advanced glycation endproducts induce fibrogenic activity in nonalcoholic steatohepatitis by modulating TNF- $\alpha$ converting enzyme activity in mice," Hepatology, vol. 58 , no. 4 , pp. 1339-1348, 2013.

[25] S. de Minicis, E. Seki, Y.-H. Paik et al., "Role and cellular source of nicotinamide adenine dinucleotide phosphate oxidase in hepatic fibrosis," Hepatology, vol. 52, no. 4, pp. 1420-1430, 2010.

[26] R. S. Tacke, H.-C. Lee, C. Goh et al., "Myeloid suppressor cells induced by hepatitis $\mathrm{C}$ virus suppress $\mathrm{T}$-cell responses through the production of reactive oxygen species," Hepatology, vol. 55, no. 2, pp. 343-353, 2012.

[27] R. Reinehr, S. Becker, A. Eberle, S. Grether-Beck, and D. Häussinger, "Involvement of NADPH oxidase isoforms and Src family kinases in CD95-dependent hepatocyte apoptosis," The Journal of Biological Chemistry, vol. 280, no. 29, pp. 2717927194, 2005.

[28] P. Sancho, J. Mainez, E. Crosas-Molist et al., "NADPH oxidase NOX4 mediates stellate cell activation and hepatocyte cell death during liver fibrosis development," PLoS ONE, vol. 7, no. 9, Article ID e45285, 2012.

[29] J. X. Jiang, X. Chen, N. Serizawa et al., "Liver fibrosis and hepatocyte apoptosis are attenuated by GKT137831, a novel
NOX4/NOX1 inhibitor in vivo," Free Radical Biology and Medicine, vol. 53, no. 2, pp. 289-296, 2012.

[30] K.-H. Kim, C.-C. Chen, R. I. Monzon, and L. F. Lau, "Matricellular protein CCN1 promotes regression of liver fibrosis through induction of cellular senescence in hepatic myofibroblasts," Molecular and Cellular Biology, vol. 33, no. 10, pp. 2078-2090, 2013.

[31] C. Bureau, J. Bernad, N. Chaouche et al., "Nonstructural 3 protein of hepatitis $C$ virus triggers an oxidative burst in human monocytes via activation of NADPH oxidase," Journal of Biological Chemistry, vol. 276, no. 25, pp. 23077-23083, 2001.

[32] F. Thorén, A. Romero, M. Lindh, C. Dahlgren, and K. Hellstrand, "A hepatitis C virus-encoded, nonstructural protein (NS3) triggers dysfunction and apoptosis in lymphocytes: role of NADPH oxidase-derived oxygen radicals," Journal of Leukocyte Biology, vol. 76, no. 6, pp. 1180-1186, 2004.

[33] N. S. R. de Mochel, S. Seronello, S. H. Wang et al., "Hepatocyte $\mathrm{NAD}(\mathrm{P}) \mathrm{H}$ oxidases as an endogenous source of reactive oxygen species during hepatitis C virus infection," Hepatology, vol. 52, no. 1, pp. 47-59, 2010.

[34] J. Colmenero, R. Bataller, P. Sancho-Bru et al., "Effects of losartan on hepatic expression of nonphagocytic NADPH oxidase and fibrogenic genes in patients with chronic hepatitis C," American Journal of Physiology-Gastrointestinal and Liver Physiology, vol. 297, no. 4, pp. G726-G734, 2009.

[35] S. De Minicis and D. A. Brenner, "Oxidative stress in alcoholic liver disease: role of NADPH oxidase complex," Journal of Gastroenterology and Hepatology, vol. 23, no. 1, pp. S98-S103, 2008.

[36] H. Kono, I. Rusyn, M. Yin et al., "NADPH oxidase-derived free radicals are key oxidants in alcohol-induced liver disease," The Journal of Clinical Investigation, vol. 106, no. 7, pp. 867-872, 2000.

[37] M. D. Wheeler, H. Kono, M. Yin et al., "The role of kupffer cell oxidant production in early ethanol-induced liver disease," Free Radical Biology and Medicine, vol. 31, no. 12, pp. 1544-1549, 2001.

[38] F. J. Cubero and N. Nieto, "Ethanol and arachidonic acid synergize to activate Kupffer cells and modulate the fibrogenic response via tumor necrosis factor $\alpha$, reduced glutathione, and transforming growth factor $\beta$-dependent mechanisms," Hepatology, vol. 48, no. 6, pp. 2027-2039, 2008.

[39] V. Thakur, M. T. Pritchard, M. R. McMullen, Q. Wang, and L. E. Nagy, "Chronic ethanol feeding increases activation of NADPH oxidase by lipopolysaccharide in rat Kupffer cells: Role of increased reactive oxygen in LPS-stimulated ERK1/2 activation and TNF- $\alpha$ production," Journal of Leukocyte Biology, vol. 79, no. 6, pp. 1348-1356, 2006.

[40] I. Levin, J. Petrasek, and G. Szabo, "The presence of p47phox in liver parenchymal cells is a key mediator in the pathogenesis of alcoholic liver steatosis," Alcoholism: Clinical and Experimental Research, vol. 36, no. 8, pp. 1397-1406, 2012.

[41] S. Yeligar, H. Tsukamoto, and V. K. Kalra, "Ethanol-induced expression of ET-1 and ET-BR in liver sinusoidal endothelial cells and human endothelial cells involves hypoxia-inducible factor-1 $\alpha$ and microRNA-199," The Journal of Immunology, vol. 183, no. 8, pp. 5232-5243, 2009.

[42] A. E. Feldstein and S. M. Bailey, "Emerging role of redox dysregulation in alcoholic and nonalcoholic fatty liver disease," Antioxidants and Redox Signaling, vol. 15, no. 2, pp. 421-424, 2011. 
[43] C. Guichard, R. Moreau, D. Pessayre, T. K. Epperson, and K.H. Krause, "NOX family NADPH oxidases in liver and in pancreatic islets: a role in the metabolic syndrome and diabetes?" Biochemical Society Transactions, vol. 36, no. 5, pp. 920-929, 2008.

[44] T. Adachi, H. Togashi, A. Suzuki et al., "NAD(P)H oxidase plays a crucial role in PDGF-induced proliferation of hepatic stellate cells," Hepatology, vol. 41, no. 6, pp. 1272-1281, 2005.

[45] R. Sugimoto, M. Enjoji, M. Kohjima et al., "High glucose stimulates hepatic stellate cells to proliferate and to produce collagen through free radical production and activation of mitogen-activated protein kinase," Liver International, vol. 25, no. 5, pp. 1018-1026, 2005.

[46] M. Carmiel-Haggai, A. I. Cederbaum, and N. Nieto, "A high-fat diet leads to the progression of non-alcoholic fatty liver disease in obese rats," FASEB Journal, vol. 19, no. 1, pp. 136-138, 2005.

[47] S. Chatterjee, D. Ganini, E. J. Tokar et al., "Leptin is key to peroxynitrite-mediated oxidative stress and Kupffer cell activation in experimental non-alcoholic steatohepatitis," Journal of Hepatology, vol. 58, no. 4, pp. 778-784, 2013.

[48] J. X. Jiang, K. Mikami, V. H. Shah, and N. J. Torok, "Leptin induces phagocytosis of apoptotic bodies by hepatic stellate cells via a Rho guanosine triphosphatase-dependent mechanism," Hepatology, vol. 48, no. 5, pp. 1497-1505, 2008.

[49] S. de Minicis, E. Seki, C. Oesterreicher, B. Schnabl, R. F. Schwabe, and D. A. Brenner, "Reduced nicotinamide adenine dinucleotide phosphate oxidase mediates fibrotic and inflammatory effects of leptin on hepatic stellate cells," Hepatology, vol. 48, no. 6, pp. 2016-2026, 2008.

[50] I. García-Ruiz, E. Gómez-Izquierdo, T. Díaz-Sanjuán et al., “Sp1 and $\mathrm{Sp} 3$ transcription factors mediate leptin-induced collagen $\alpha 1$ (I) gene expression in primary culture of male rat hepatic stellate cells," Endocrinology, vol. 153, no. 12, pp. 5845-5856, 2012.

[51] H. Vlassara and G. E. Striker, "AGE restriction in diabetes mellitus: a paradigm shift," Nature Reviews Endocrinology, vol. 7, no. 9, pp. 526-539, 2011.

[52] A. dela Peña, I. A. Leclercq, J. Williams, and G. C. Farrell, "NADPH oxidase is not an essential mediator of oxidative stress or liver injury in murine MCD diet-induced steatohepatitis," Journal of Hepatology, vol. 46, no. 2, pp. 304-313, 2007.

[53] A. E. Feldstein, A. Canbay, P. Angulo et al., "Hepatocyte apoptosis and fas expression are prominent features of human nonalcoholic steatohepatitis," Gastroenterology, vol. 125, no. 2, pp. 437-443, 2003.

[54] J. H. Yu, B.-M. Zhu, G. Riedlinger, K. Kang, and L. Hennighausen, "The liver-specific tumor suppressor STAT5 controls expression of the reactive oxygen species-generating enzyme NOX4 and the proapoptotic proteins PUMA and BIM in mice," Hepatology, vol. 56, no. 6, pp. 2375-2386, 2012.

[55] Y.-H. Paik, J. Kim, T. Aoyama, S. De Minicis, R. Bataller, and D. A. Brenner, "Role of NADPH oxidases in liver fibrosis," Antioxidants and Redox Signaling, vol. 20, no. 17, pp. 2854-2872, 2014.

[56] R. Bataller, R. F. Schwabe, Y. H. Choi et al., "NADPH oxidase signal transduces angiotensin II in hepatic stellate cells and is critical in hepatic fibrosis," Journal of Clinical Investigation, vol. 112, no. 9, pp. 1383-1394, 2003.

[57] R. K. Ambasta, P. Kumar, K. K. Griendling, H. H. H. W. Schmidt, R. Busse, and R. P. Brandes, "Direct interaction of the novel Nox proteins with p22phox is required for the formation of a functionally active NADPH oxidase," Journal of Biological Chemistry, vol. 279, no. 44, pp. 45935-45941, 2004.

[58] I. Carmona-Cuenca, C. Roncero, P. Sancho et al., "Upregulation of the NADPH oxidase NOX4 by TGF-beta in hepatocytes is required for its pro-apoptotic activity," Journal of Hepatology, vol. 49, no. 6, pp. 965-976, 2008.

[59] T. Aoyama, Y. H. Paik, S. Watanabe et al., "Nicotinamide adenine dinucleotide phosphate oxidase in experimental liver fibrosis: GKT137831 as a novel potential therapeutic agent," Hepatology, vol. 56, no. 6, pp. 2316-2327, 2012.

[60] S. Senturk, M. Mumcuoglu, O. Gursoy-Yuzugullu, B. Cingoz, K. C. Akcali, and M. Ozturk, "Transforming growth factorbeta induces senescence in hepatocellular carcinoma cells and inhibits tumor growth," Hepatology, vol. 52, no. 3, pp. 966-974, 2010.

[61] E. Crosas-Molist, E. Bertran, P. Sancho et al., "The NADPH oxidase NOX4 inhibits hepatocyte proliferation and liver cancer progression," Free Radical Biology \& Medicine, vol. 69, pp. 338347,2014

[62] P. Sancho and I. Fabregat, "NADPH oxidase NOX1 controls autocrine growth of liver tumor cells through up-regulation of the epidermal growth factor receptor pathway," Journal of Biological Chemistry, vol. 285, no. 32, pp. 24815-24824, 2010.

[63] M. Daugaard, R. Nitsch, B. Razaghi et al., "Hacel controls ROS generation of vertebrate Racl-dependent NADPH oxidase complexes," Nature Communications, vol. 4, article 2180, 2013.

[64] C. D. Klaassen and S. A. Reisman, "Nrf2 the rescue: effects of the antioxidative/electrophilic response on the liver," Toxicology and Applied Pharmacology, vol. 244, no. 1, pp. 57-65, 2010.

[65] S. M. Shin, J. H. Yang, and S. H. Ki, "Role of the Nrf2-are pathway in liver diseases," Oxidative Medicine and Cellular Longevity, vol. 2013, Article ID 763257, 9 pages, 2013.

[66] S. Schaedler, J. Krause, K. Himmelsbach et al., "Hepatitis B virus induces expression of antioxidant response element-regulated genes by activation of Nrf2," The Journal of Biological Chemistry, vol. 285, no. 52, pp. 41074-41086, 2010.

[67] D. Burdette, M. Olivarez, and G. Waris, "Activation of transcription factor Nrf2 by hepatitis $\mathrm{C}$ virus induces the cell-survival pathway," Journal of General Virology, vol. 91, no. 3, pp. 681-690, 2010.

[68] K. C. Wu, J. Liu, and C. D. Klaassen, "Role of Nrf2 in preventing ethanol-induced oxidative stress and lipid accumulation," Toxicology and Applied Pharmacology, vol. 262, no. 3, pp. 321-329, 2012.

[69] J. Lamlé, S. Marhenke, J. Borlak et al., "Nuclear factor-eythroid 2-related factor 2 prevents alcohol-induced fulminant liver injury," Gastroenterology, vol. 134, no. 4, pp. 1159.e2-1168.e2, 2008.

[70] J. Liu, K. C. Wu, Y.-F. Lu, E. Ekuase, and C. D. Klaassen, "NRF2 protection against liver injury produced by various hepatotoxicants," Oxidative Medicine and Cellular Longevity, vol. 2013, Article ID 305861, 8 pages, 2013.

[71] H. Sugimoto, K. Okada, J. Shoda et al., "Deletion of nuclear factor-E2-related factor-2 leads to rapid onset and progression of nutritional steatohepatitis in mice," American Journal of Physiology: Gastrointestinal and Liver Physiology, vol. 298, no. 2, pp. G283-G294, 2010.

[72] S. Chowdhry, M. H. Nazmy, P. J. Meakin et al., "Loss of Nrf2 markedly exacerbates nonalcoholic steatohepatitis," Free Radical Biology and Medicine, vol. 48, no. 2, pp. 357-371, 2010. 
[73] R. Shimozono, Y. Asaoka, Y. Yoshizawa et al., "Nrf2 activators attenuate the progression of nonalcoholic steatohepatitisrelated fibrosis in a dietary rat model," Molecular Pharmacology, vol. 84, no. 1, pp. 62-70, 2013.

[74] U. A. Kohler, S. Kurinna, D. Schwitter et al., "Activated Nrf2 impairs liver regeneration in mice by activation of genes involved in cell cycle control and apoptosis," Hepatology, vol. 60, no. 2, pp. 670-678, 2013.

[75] L. Hecker, N. J. Logsdon, D. Kurundkar et al., "Reversal of persistent fibrosis in aging by targeting Nox4-Nrf2 redox imbalance," Science Translational Medicine, vol. 6, Article ID 231ra247, 2014.

[76] I. Tikhanovich, J. Cox, and S. A. Weinman, "Forkhead box class O transcription factors in liver function and disease," Journal of Gastroenterology and Hepatology, vol. 28, no. 1, pp. 125-131, 2013.

[77] D. R. Calnan and A. Brunet, "The FoxO code," Oncogene, vol. 27, no. 16, pp. 2276-2288, 2008.

[78] S. Nemoto and T. Finkel, "Redox regulation of forkhead proteins through a p66shc-dependent signaling pathway," Science, vol. 295, no. 5564, pp. 2450-2452, 2002.

[79] M. Adachi, Y. Osawa, H. Uchinami, T. Kitamura, D. Accili, and D. A. Brenner, "The forkhead transcription factor FoxO1 regulates proliferation and transdifferentiation of hepatic stellate cells," Gastroenterology, vol. 132, no. 4, pp. 1434-1446, 2007.

[80] M. Matsumoto, S. Han, T. Kitamura, and D. Accili, "Dual role of transcription factor FoxO1 in controlling hepatic insulin sensitivity and lipid metabolism," The Journal of Clinical Investigation, vol. 116, no. 9, pp. 2464-2472, 2006.

[81] V. S. Calvert, R. Collantes, H. Elariny et al., "A systems biology approach to the pathogenesis of obesity-related nonalcoholic fatty liver disease using reverse phase protein microarrays for multiplexed cell signaling analysis," Hepatology, vol. 46, no. 1, pp. 166-172, 2007.

[82] L. Deng, I. Shoji, W. Ogawa et al., "Hepatitis C virus infection promotes hepatic gluconeogenesis through an NS5A-mediated, FoxO1-dependent pathway," Journal of Virology, vol. 85, no. 17, pp. 8556-8568, 2011.

[83] G. Z. Tao, N. Lehwald, K. Y. Jang et al., "Wnt/ $\beta$-catenin signaling protects mouse liver against oxidative stress-induced apoptosis through the inhibition of forkhead transcription factor FoxO3," Journal of Biological Chemistry, vol. 288, no. 24, pp. 17214-17224, 2013. 


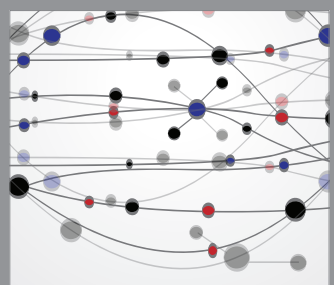

The Scientific World Journal
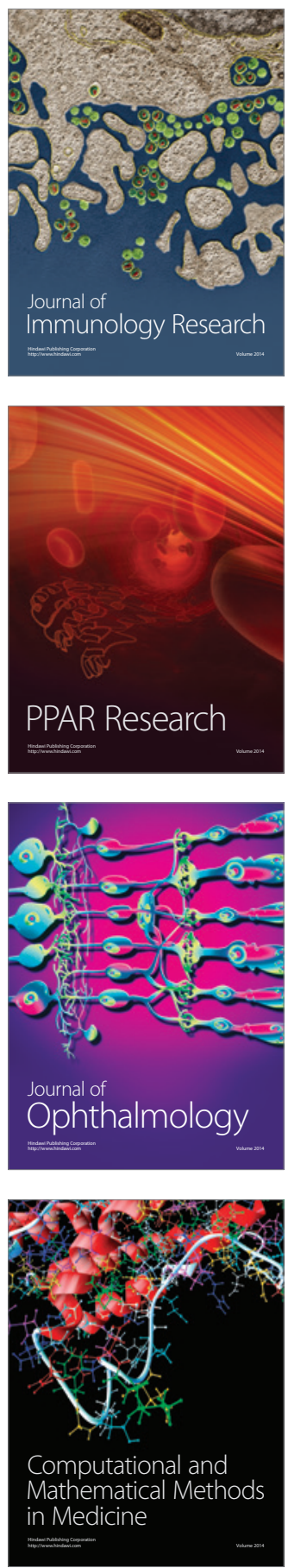

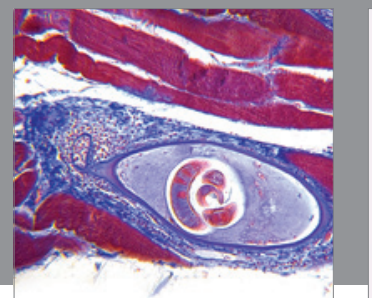

Gastroenterology

Research and Practice
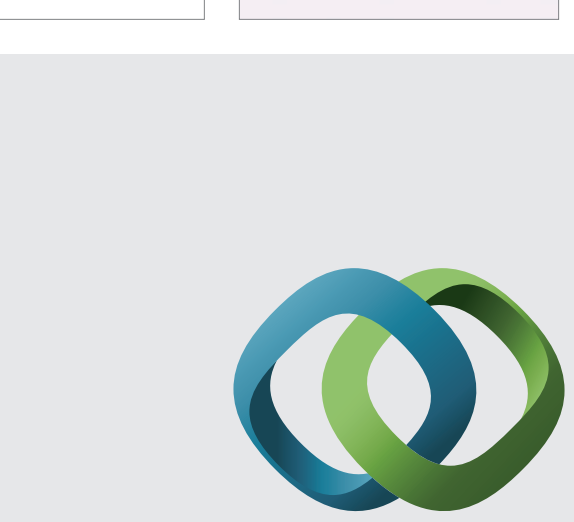

\section{Hindawi}

Submit your manuscripts at

http://www.hindawi.com
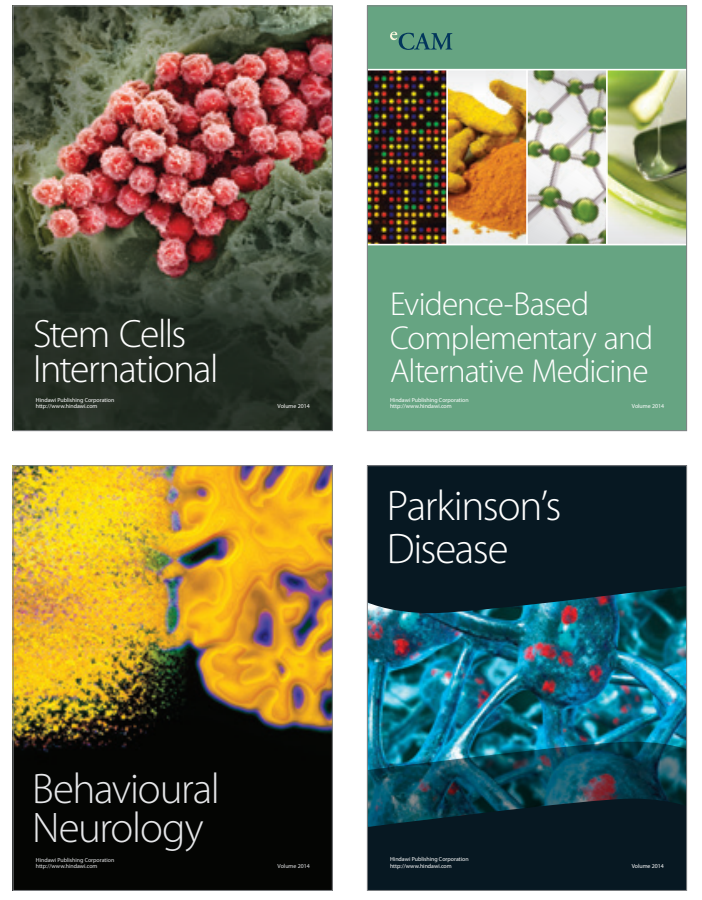
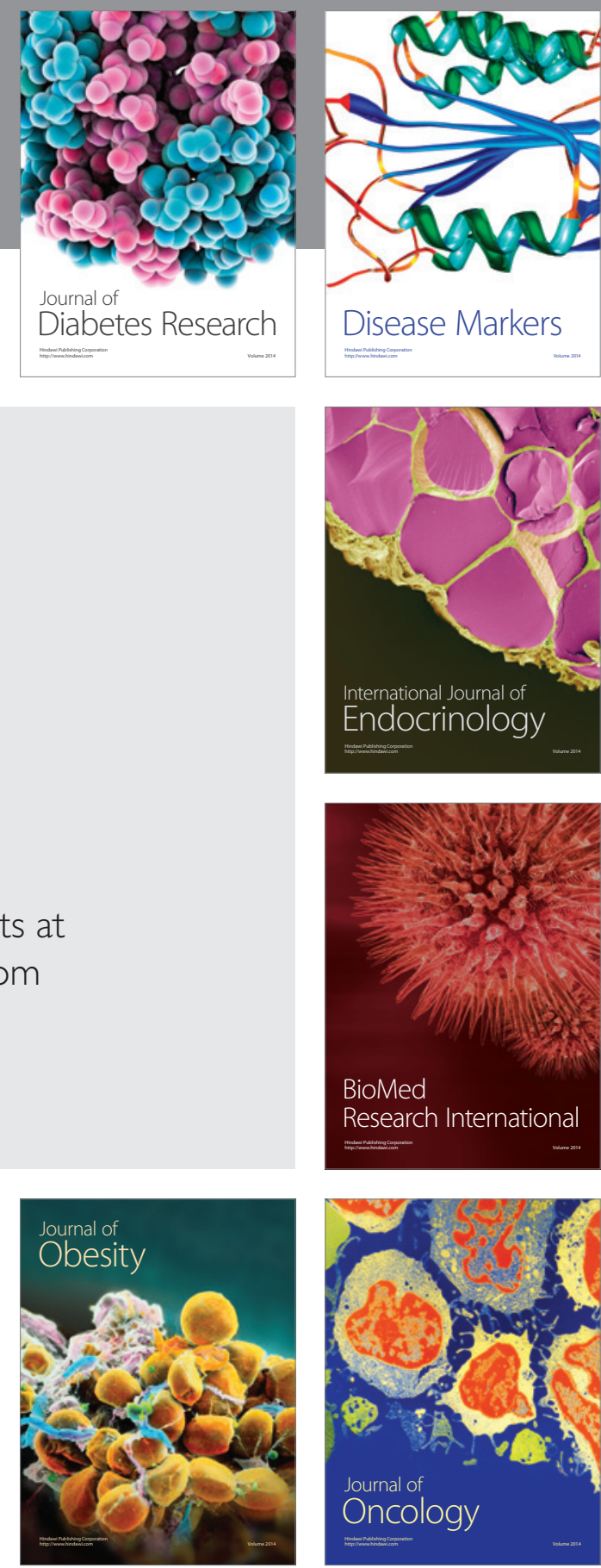

Disease Markers
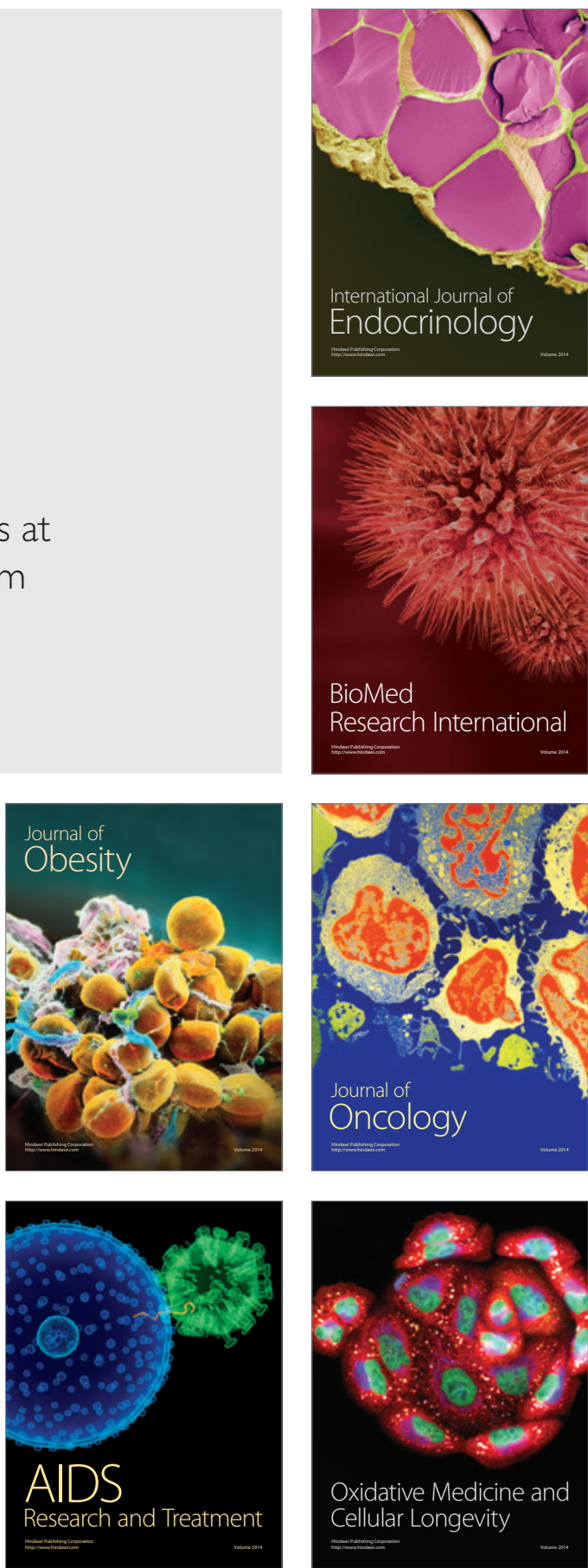